\title{
Un proyecto experimental para la protección y puesta en valor del patrimonio arqueológico subacuático: el Proyecto Tarifa
}

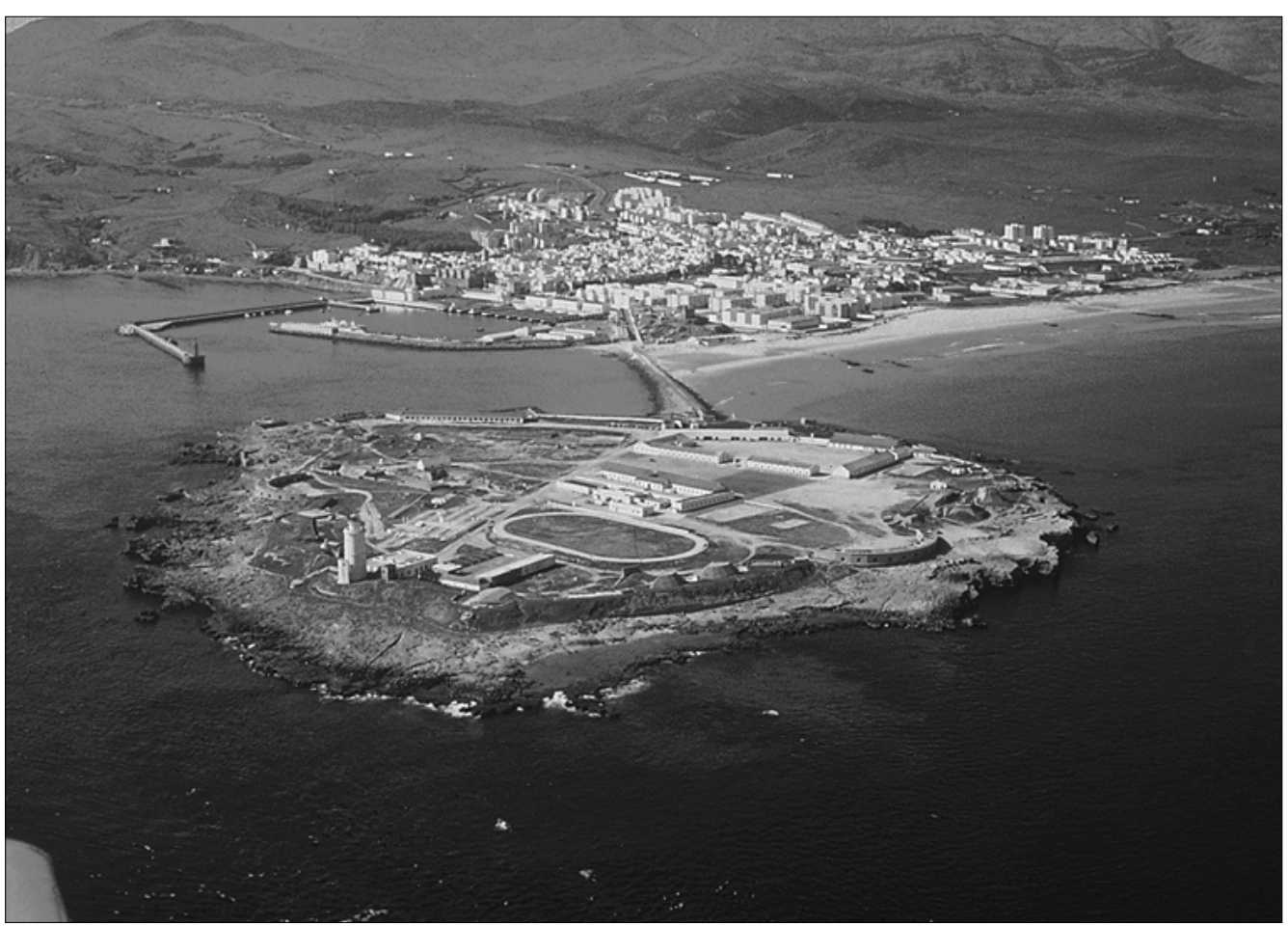

Carlos Alonso Villalobos María Navarro Domínguez

Departamento de Documentación y Protección Centro de Arqueología Subacuática. IAPH

\section{Resumen}

La gran riqueza del patrimonio histórico subacuático, el alto índice de actuaciones extractivas ilícitas realizadas sobre objeto del mismo, y la fuerte amenaza realizada que supone un creciente y descontrolado sector turístico-deportivo relacionado con la actividad subacuática, son factores que han servido para calificar el ámbito litoral de Tarifa como zona de alto riesgo para nuestros bienes culturales sumergido. Experimentalmente, para investigar en la búsqueda de técnicas y métodos aplicados a la protección de estos bienes, se diseña el Proyecto Tarifa, en el que patrimonio cultural y natural, con sus diferentes recursos, se unen dentro de una misma oferta de protección, puesta en valor y transmisión social.

\section{Palabras claves}

Protección, patrimonio arqueológico subacuático, Tarifa, puesta en valor

La estrecha relación que Andalucía ha mantenido con el mar a lo largo de la historia, y las excepcionales condiciones de conservación que se pueden llegar a dar en el medio subacuático, han permitido que bajo nuestras aguas exista una excepcional riqueza cultural (pecios, restos portuarios y constructivos, fondeaderos, etc.). 
Cada uno de los yacimientos que componen este $\mathrm{Pa}$ trimonio Arqueológico Subacuático (PAS) son un ejemplo único e irrepetible. Un barco y su carga son cronológica y culturalmente un conjunto cerrado y homogéneo donde todos los elementos están íntimamente relacionados en un perfecto sincronismo.

Las excepcionales condiciones físico-químicas que se dan en el medio ambiente subacuático, permiten la estabilidad de restos materiales y orgánicos que no se han llegado a conservar en los registros arqueológico terrestres. Gracias a ello los técnicos pueden reconstruir partes de nuestra historia sólo conservada en los yacimientos subacuáticos. La destrucción de este patrimonio supone perder, para siempre, la posibilidad de reconstruir esos momentos del pasado, por ello esta importantísima parte de nuestros bienes culturales -el patrimonio histórico sumergido- es, también, un recurso a proteger.

La creación del Centro de Arqueología Subacuática (CAS) del Instituto Andaluz del Patrimonio Histórico (IAPH) (VV. AA., 1999: 109-137.) supuso en este sentido un punto de inflexión en la política de tutela del patrimonio andaluz. Condicionantes como la supuesta "inaccesibilidad" al medio hiperbárico, la escasez de técnicos especialistas en los diferentes campos de la tutela del patrimonio arqueológico subacuático, y la falta de una visión clara sobre la problemática específica de este patrimonio, sirvieron, hasta ese momento, como discurso para dar la espalda al patrimonio histórico sumergido. La gestación del CAS supuso, administrativamente, el reconocimiento de ese rico potencial cultural hasta el momento olvidado. No obstante, cambiar la situación anterior y mejorar el nivel de conocimiento hasta alcanzar niveles óptimos en la gestión y tutela del mismo, requiere años de trabajo técnico y altos niveles de inversión.

El retraso de la investigación arqueológica subacuática española en general y andaluza en particular, frente a la de otros países y/o comunidades (Nieto, X., 1999 : 138); el gran desconocimiento existente sobre el potencial, diversidad y realidad de nuestro patrimonio cultural sumergido; la falta de técnicos especialistas en los diferentes campos de su tutela; la inexistencia, en nuestra Comunidad, de una línea de investigación específica que se ocupe de este patrimonio; la imperante y generalizada visión aventurera y de "caza tesoros" que se vincula a este mundo, y que, sobre la base de una profunda ignorancia acerca de la problemática del patrimonio sumergido ', aún está presente en muchos ámbitos de nuestra sociedad, son retos que deben ir superándose.

Las directrices culturales para el futuro deberán hacerse eco de ello. En este sentido se ha manifestado la UNESCO (ver al respecto Pérez del Campo, L., 1999.), planteando a los estados ribereños de océanos, mares y aguas interiores, recomendaciones específicas encaminadas a mejorar la protección del patrimonio histórico sumergido frente a la amenaza de una explotación ilícita y ante la debilidad de los actuales instrumentos legales de protección.

\section{Protección y puesta en valor del patrimonio arqueológico subacuático}

Los múltiples riesgos a que está sometido nuestro patrimonio y la necesidad de garantizar su conservación, son las premisas que marcan las prioridades y directrices de intervención sobre el mismo. Sin embargo, la disponibilidad de recursos económicos es la verdadera encargada de dictar la realidad y el alcance de esas intervenciones (Caravaca, I., et al., 1997.). En esta coyuntura se pone de manifiesto que, políticas culturales basadas únicamente en los recursos del Estado como forma de garantizar la preservación, no son válidas para la realidad socioeconómica en que vivimos. Es preciso buscar nuevas fórmulas de gestión patrimonial "abriendo" el patrimonio a la participación de todos aquellos agentes sociales que puedan colaborar en las labores de documentación, protección, intervención, conservación y difusión del mismo. En este sentido las nuevas tendencias de actuación y gestión en el campo del Patrimonio Cultural (Fernández-Baca, R. 1999.) ponen de manifiesto la imperiosa necesidad de mejorar el vínculo entre Patrimonio y Sociedad, atendiendo las demandas de los diferentes colectivos y agentes sociales vinculados a él sobre la base de su territorialidad y uso. En esta misma línea, el Plan General de Bienes Culturales (PGBC) de la Consejería de Cultura para su segundo periodo de vigencia (1996-2000), marca entre sus prioridades como línea a seguir, promover el uso y disfrute sostenible del patrimonio como forma de incrementar la calidad de vida de los andaluces en los diversos ámbitos territoriales de nuestra Comunidad.

Desde el departamento de documentación y protección del CAS del IAPH, siguiendo el concepto de sostenibilidad marcado por la Conferencia de las Naciones Unidas sobre el Medio Ambiente y el Desarrollo (CNUMA $)^{2}$, se viene trabajando, sobre la base de los principios de interacción y sostenibilidad, en la búsqueda de herramientas más eficaces para la protección y puesta en valor del patrimonio cultural sumergido.

Como recoge el PGBC, el Patrimonio Histórico, y cualquier política que se establezca sobre el mismo, "no puede ser entendido si no es en su dimensión territorial y en su relación con su entorno natural y social en el que se encuadra". Es importante por ello actuar sobre el hecho cultural simultáneamente al socioeconómico, y nunca de espaldas a él; y es preciso por tanto, dado que sólo se conserva aquello que se usa ${ }^{3}$, convertir los "pasivos patrimoniales" en activos sociales productivos, para obtener retroactivamente garantía de preservación de los bienes.

En este sentido, tomando el territorio litoral como base física sobre la que implementar otras políticas sectoriales de desarrollo, y aplicando fórmulas de gestión integral, creemos posible diseñar herramientas más eficaces de protección, armonizando simultáneamente el hecho cultural y el desarrollo sectorial y regional. Con esta filosofía de gestión, desde el departamento de documentación y protección del CAS, se viene trabajando específicamente en la búsqueda de herramientas eficaces para la protección y puesta en valor del patrimonio

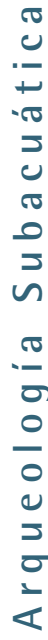

I. Obsérvese al respecto el matiz aventurero con que, los diferentes medios de comunicación social, difunden noticias referentes al patrimonio subacuático. En parte, el origen, habría que buscarlo en la constante imitación de las series documentales $y$ modelos de información norteamericanos, un país en el que la legislación, sin reconocer sus valores patrimoniales, permite la extracción de los "tesoros" contenidos en los antiguos barcos hundidos. La realidad europea y española en materia de protección de patrimonio es, afortunadamente, bien diferente. No obstante existen determinados colectivos que, ignorando la realidad del PAS y las recomendaciones técnicas -planteadas incluso en informes internacionalesproponen a la comunidad y la Administración la necesidad de ejecutar grandes proyectos de recuperación de pecios detrás de los cuales, en múltiples casos, se esconden altos beneficios para empresa especializadas

2. La CNUMA entiende por desarrollo sostenible, todo aquel proceso que permite el desarrollo sin maltratar o agotar los recursos que lo hacen posible. CITA libro BARRAGÁN.).

3. Cita Libro Patrimonio Cultural y Sociedad. 


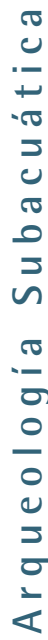

cultural sumergido, compatibilizando cuatro elementos diferentes pero interrelacionados: patrimonio cultural subacuático, medioambiente, turismo y deporte.

\section{EI Proyecto Tarifa}

El Proyecto Tarifa es una primera propuesta, planteada a un nivel experimental y microespacial (la Isla de Tarifa) en la que se aplican las directrices de gestión cultural ya apuntadas. Con su formulación se pretende investigar en el diseño de nuevas formas de protección para nuestro patrimonio cultural sumergido, utilizando experiencias de otros países y adaptando modelos ya empleados en otros ámbitos.

La elección de Tarifa y su entorno viene justificada por darse en él tres premisas básicas:

- Gran riqueza patrimonial subacuática -en buena medida desconocida-.

- Intensa y creciente presión turística y deportiva que repercute sobre el patrimonio subacuático.

- Alto índice de actividades extractivas ilícitas realizadas sobre bienes del patrimonio sumergido.

\section{Objetivos específicos}

Los objetivos específicos que se persiguen en las diferentes fases del desarrollo del Proyecto son:

- Documentar la riqueza del PAS de la zona, analizando su diversidad y evaluando los riesgos a que está sometido.

- Investigar en la mejora de los métodos y técnicas aplicados para su tutela, garantizando la protección de los mismos y buscando un mayor grado de corresponsabilidad en los diferentes niveles de la administración.

- Concienciar acerca de la fragilidad del PAS y el fuerte daño que el expolio produce sobre él.

- Incorporar los Bienes Culturales a la dinámica social y productiva, mostrando a los diferentes sectores sociales, especialmente a los vinculados con el buceo, las enormes posibilidades que un correcto uso del PAS puede aportar como generador de empleo y riquezas.

4. La Ley $|/| 99 \mid$ de

Patrimonio Histórico

Andaluz (LPHA), en sus artículos 48 y 49, define el concepto de zona de servidumbre arqueológica como ".. aquellos espacios claramente determinados en que se presupone fundadamente la existencia de restos arqueológicos de interés y se considera necesario adoptar medidas precautorias.".
- Implicar a estos colectivos en labores de protección.

\section{Propuesta de protección}

La riqueza del PAS en el ámbito marino de Tarifa es tan amplia que resulta de vital importancia establecer medidas para su protección. Este hecho, que no debiera entrañar demasiados problemas con la vigente legislación de patrimonio, en cuyo articulado se re- cogen figuras específicas diseñadas al respecto, se muestra insuficiente cuando el bien cultural a proteger se encuentra situado en el medio subacuático.

En materia de PAS, el primer paso lógico, que sería el conocimiento del bien a proteger para establecer posteriores premisas de actuación, no es el camino idóneo. Aunque en la actualidad este patrimonio está siendo cada vez más objeto de investigación, en su mayoría está compuesto por restos, zonas y/o yacimientos arqueológicos -visibles o no, y/o localizados o no-, que, aunque conocidos, aún no han podido ser valorados históricamente. Son estos los que deben ser protegidos con mayor rigor, pues su conservación no sólo supone poder llegar al conocimiento de un proceso histórico, sino además ofrece la posibilidad de no comprometer el futuro de nuevas generaciones de arqueólogos, premisa esta fundamental si aceptamos definitivamente el concepto sostenibilidad para el bien cultural. Si bien la Ley de Patrimonio Histórico de Andalucía ( $\mathrm{LPHA}$ ) acuña el término zona de servidumbre arqueológica para definir estos casos ${ }^{4}$, la figura se muestra insuficiente, no habiéndose utilizado hasta el presente por el somero blindaje que hace de los yacimientos frente a la actuación antrópica. Es preciso pues trabajar en este campo adaptando la legislación vigente, basada en normativas y reglamentaciones territoriales, y estableciendo de manera urgente estrategias prácticas y eficaces que permitan alcanzar la deseada protección de nuestro patrimonio sumergido. En esta línea, el proyecto Tarifa pretende ir más allá de la idea de una administración pública como sola responsable de esta tutela del patrimonio, investigando para ello sobre la eficacia de fórmulas de protección del patrimonio sumergido ya experimentadas en otros países y especialmente para el ámbito del patrimonio natural.

Como estrategia de actuación se ha considerado apropiado investigar en un doble campo:

- La aplicación de herramientas de protección espacial para controla el acceso y uso público del espacio protegido y de sus bienes, utilizando los recursos que ofrecen tanto la legislación de patrimonio como la de medio ambiental.

- La puesta en valor de estos espacios y su patrimonio como forma de difundir, concienciar y vincular en su protección a los distintos agentes sociales.

La aplicación a escala local de esta formula de protección permitiría, además, concienciar a los diferentes colectivos sociales acerca de la riqueza, variedad, fragilidad del PAS, haciéndoles ver la capacidad generadora de riquezas que un correcto uso del mismo puede tener. Este hecho, que conlleva en primera instancia un arduo trabajo de sensibilización pública en el que la Administración debe dar el primer paso, pasa por la premisa de habilitar fórmulas de compromiso con los diferentes colectivos sociales de cara a la conservación del bien cultural en uso.

La coincidencia espacial y temporal de nuestro proyecto con el Plan de Ordenación de los Recursos Natu- 
rales (PORN) del frente litoral Algeciras-Tarifa 5 , promovido por la Consejería de Medio Ambiente de la Junta de Andalucía, ha servido para unificar criterios en el diseño de estrategias de actuación conjunta para el patrimonio cultural y natural. En este sentido, a lo largo de 1999 se viene trabajando conjuntamente con el equipo encargado de redactar el PORN 6 , habiéndose conseguido que por vez primera una normativa ambiental, incluya, reconozca y proteja como recurso cultural al patrimonio histórico sumergido. Además, la creación en un futuro próximo de una figura específica de protección ambiental para el espacio litoral Algeciras-Tarifa, va a permitir y agilizar el control sobre el acceso a los diferentes recursos naturales y culturales.

\section{Fases de actuación}

Para la consecución de los objetivos marcados, el Proyecto se ha dividido en cuatro fases independientes pero íntimamente relacionadas entre sí: documentación, intervención, interpretación y puesta en valor. El PORN, no obstante, ha venido a plantear la necesidad de alterar la programación inicialmente prevista dada la necesidad a corto plazo de atender un marco espacil más amplio -zona comprendida entre Cabo Gracia y Punta Carnero- del inicialmente previsto (Isla de Tarifa.).

\section{Fase de Documentación}

El objetivo de esta primera fase, en la que actualmente se trabaja, es doble:

- Identificar los diferentes registros arqueológicos que componen el PAS dentro de la zona de actuación del proyecto, sistematizando la información existente y determinando el grado de riesgo a que están sometidos.

- Realizar un estudio comparativo sobre diferentes experiencias para la puesta en valor de espacios culturales subacuáticos, atendiendo al diseño didáctico de los mismos y a la especificidad de las fórmulas de protección y vigilancia utilizadas.

En ambos casos es preciso partir de un ámplio estudio de investigación documental. Para Tarifa contamos con la existencia de un trabajo anterior realizado desde el I.A.P.H. en el que se recoge y analiza la información aportada para el conocimiento del PAS por diferentes tipos de fuentes documentales (Martí, J., 1997).

Tomando como base este trabajo, y al objeto de profundizar más en la recopilación de documentación oral y vincular desde un primer momento al proyecto a los clubes de buceo y deportistas del entorno de Tarifa, agentes sociales estrechamente vinculados a los objetivos que se persiguen, se han mantenido en la sede de CAS reuniones técnicas con estos colectivos. En ellas se les ha informado del proyecto, abriendoles la posibilidad de colaborar en las labores de documentación y localización de los registros arqueológicos conocidos. Cada uno de los yacimientos identificados está siendo objeto de posicionamiento, registrándose gráficamente por distintos medios, y realizándose sobre ellos una primera valoración de su importancia histórico-arqueológica, estado de conservación y factores de riesgo que puedan incidir sobre ellos.

Simultáneamente al desarrollo de los trabajos subacuáticos, contemplado tanto en esta primera fase como en la siguiente, se viene trabajando en la que consideramos columna vertebral del proyecto: el diseño de las herramientas a utilizar para la puesta en valor, garantizando la preservación del PAS, promoviendo el uso respetuoso y sostenible de estos bienes culturales, y perfeccionando los instrumentos de tutela.

\section{Fase de intervención}

En el entorno de la Isla de Tarifa, como consecuencia del importante papel jugado por esta zona geográfica a lo largo de las diferentes etapas históricas, existen sin duda muchos otros yacimientos aún por concer y documentar. Al objeto de proceder a su localización, desde el C.A.S. se ha programado una amplia campaña de prospección en la que se conjugan tanto técnicas de teledetección como de prospección visual.

Dentro de esta fase está previsto intervenir arqueológicamente sobre determinados yacimientos seleccionados apriori para su puesta en valor por las altas posibilidades que ofrecen de cara a una correcta difusión y el escaso riesgo que plantea su uso. Los sistemas y métodos de trabajo a emplear se adecuarán en todo momento a las exigencias y características del fondo y la profundidad, variables que marcan las condiciones específicas de actuación en un marco litoral en el que muy cerca de la costa se alcanzan cotas de profundidad superiores a los $40 \mathrm{~m}$.

\section{Fase de interpretación}

Documentados los yacimientos por los distintos medios que la tecnología pone hoy a nuestro alcance, y realizadas las intevenciones arqueológicas que se consideren de interés para su valoración, se procederá al análisis de los datos obtenidos y a la redacción de la oportunas memorias cientifico-técnicas. En ella se recogerá, además de la correspondiente síntesis interpretativa, una evaluación del riesgo a que se pueden ven sometidos los yacimientos, y propuestas técnicas a adoptar para garantizar la conservación y protección de los mismos.

Analizados los diferentes modelos y experiencias utilizados en otros paises se planteará una propuesta técnica para la puesta en valor del patrimonio subacuático en el ambito de acción del Proyecto. En ella se recogerán:

- propuestas específicas de protección legal y/o física de los yacimientos

- diseño de itinerarios para la visita subacuática guiada de zonas atendiendo a los valores culturales y naturales de las mismas

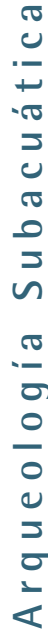

5. Acuerdo de 9 de febrero de 1999. Boletín Oficial de la Junta de Andalucía (BOJA), 25: 25।8-2521.

6. A lo largo de 1999 se ha venido colaborando con el equipo encargado, por la Consejería de Medio Ambiente de la Junta de Andalucía, de la redacción del Proyecto "Criterios técnicos para la ordenación de los recursos naturales del frente litoral de los términos municipales AlgecirasTarifa", coordinado por D. José Carlos García, Prof. del Laboratorio de Biología Marina de la Facultad de Biológicas de la Universidad de Sevilla. 


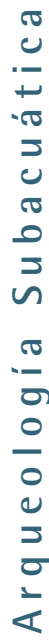

\section{Fase de difusión y puesta en valor}

Se trata sin duda de la fase más significativa del Proyecto cuya finalidad es mostrar a los diferentes agentes sociales el significado e importancia social del PAS, su necesidad de protección y la capacidad generadora de beneficios que un correcto uso del PAS puede tener mediante la correcta puesta en valor de algunos de sus bienes. Esta fase se articula através de un programa específico de difusión destinado tanto a clubes de buceo, como a los deportistas individuales que practican esta disciplina en el ámbito de Tarifa.

Una vez en desarrollo el programa de difusión y adoptadas, con garantías, fórmulas específicas para la protección que permitan controlar el acceso público al patrimonio sumergido de la zona, se iniciará a modo experimental labores de puesta en valor de algunos de estos bienes dentro de ofertas controladas. Para ello, una vez evaluados los riesgos de cada zona, se procederá al diseño de circuitos y/o itinerarios arqueológicos subacuáticos, y a las herramientas necesarias para la difusión de los mismos.

En esta etapa de puesta en valor los clubes de buceo, alcanzado un nivel óptimo de concienciación y tras una fase de cualificación, se podrían convertir en visagra para atender las demandas culturales planteadas por el sector turístico, colaborando indirecta- mente en las labores de vigilancia del patrimonio. En este sentido, para esa última fase, se contempla el diseño de una actividad didáctica-formativa específica destinada a estos colectivos siguiendo modelos ya experimentados en otros paises.

En la difusión del Proyecto Tarifa se contempla además acercar el patrimonio sumergido a sectores sociales no vinculados ni relacionados con el buceo. Exposiciónes, ciclo de conferencias y audiovisuales, jornadas de puertas abiertas del CAS y sus instalaciones, serán recursos y herramientas útiles al respecto.

\section{Conclusión}

Como pone de manifiesto en el PGBC, el patrimonio histórico y cualquier política que se establezca sobre el mismo, "no puede ser entendido si no es en su dimensión territorial y en su relación con su entorno natural y social en el que se encuadra". Un programa de protección ha de responder a este nuevo concepto de tutela, enlazando necesariamente las actuaciones de protección con otras acciones de conservación, restauración, difusión, investigación y en definitiva puesta en valor de los bienes, sin olvidar la estrecha vinculación del patrimonio histórico con el desarrollo social y económico de su entorno.

El Proyecto Tarifa, como modelo integral de actuación, pretende buscar fórmulas ágiles y directas para la protección de un patrimonio -el sumergido-, en gran medida desatendido hasta el presente, aunando todos los recursos disponibles (patrimoniales, ambientales, turísticos, etc) y aplicándolos a los diferentes campos de su gestión.

\section{Bibliografía}

CARAVACA BARROSO, I et al. ( I 997) "El patrimonio cultural como factor de desarrollo en Andalucía". Boletín del Instituto Andaluz del Patrimonio Histórico PH, 20: 87-97.

FERNÁNDEZ-BACA CASARES, R. (1999) "Patrimonio histórico, cohesión social e innovación". Boletín del Instituto Andaluz del Patrimonio Histórico PH, 27: I | 8-123.

MARTí SOLANO, J. (1997) La carta de riesgo Antrópico. Aplicación a la realidad del Patrimonio Arqueológico Subacuático de Andalucía. Provincia de Cádiz. Instituto Andaluz del Patrimonio Histórico. Centro de Arqueología Subacuática (Memoria inédita).
NIETO PRIETO, Xavier (1999): "Hacia la normalización de la arqueología subacuática en España". Boletín del Instituto Andaluz del Patrimonio Histórico, 26: 138-143.

PÉREZ DEL CAMPO, L. (1999) "Hacia una Convención Internacional para la protección del Patrimonio Histórico Subacuático". Boletín del Instituto Andaluz del Patrimonio Histórico PH, 26: 99 108.

W.AA. (1999) "Dossier". En Boletín del Instituto Andaluz del Patrimonio Histórico PH, 26: 109- 137. 